\title{
Levinson-Type Extensions for Non-Toeplitz Systems
}

\author{
Milton Porsani and Tad J. Ulrych
}

\begin{abstract}
We show that Levinson's basic principle for the solution of normal equations which are of Toeplitz form may be extended to the case where these equations do not possess this specific symmetry. The use of Levinson's principle allows us to obtain a compact $(2 \times 2)$ form to express a system of equations of arbitrary order. This compact form is the key expression in the development of recursive algorithms and allows a compact representation of the most important Levinsontype algorithms which are used in the analysis of seismic and time series data in general. In the case when the coefficient matrix does not possess any type of special structure, the number of multiplications and divisions required in the inversion is $n^{3}-2 n^{2}+4 n$. We illustrate the described method by application to various examples which we have chosen so that the coefficient matrix possesses various symmetries. Specifically, we first consider the solution of the normal equations when the associated matrix is the doubly symmetric non-Toeplitz covariance matrix. Second, we obtain the solution of extended Yule-Walker equations where the coefficient matrix is Toeplitz but nonsymmetric. Finally, we briefly illustrate the approach by considering the determination of the prediction error operator when the NE are in fact of symmetric Toeplitz form.
\end{abstract}

\section{INTRODUCTION}

L

EVINSON'S recursion (LR) [1] finds application in all Lcases where predictive or autoregressive systems are encountered. Specific examples are predictive deconvolution and maximum entropy spectral analysis. A large number of important recursive algorithms, ([2]-[6]) have as their basis the LR which linearly combines the forward and backward solutions of subsystems of lesser order. In the case when the coefficient matrix is symmetric Toeplitz, all the subsystems which occur along it's principal diagonal are equal and this fact greatly simplifies the establishment of a recursive solution. The LR approach has recently been extended to nonsymmetric Toeplitz matrices by G. Rybicki (cited in [7]) and as far as we are aware constitutes the only extension of the LR approach to matrices other than of symmetric Toeplitz form. When the coefficient matrix is no longer Toeplitz, on the other hand, subsystems of equal order are not necessarily the same and the LR cannot be applied in it's usual form. The insertion of the linear relationship which exists between solutions of lesser order directly into the quadratic form associated with the normal equations, NE, allows us to establish a form for the solution in terms of a single coefficient which must be determined. In this manner we obtain a compact $(2 \times 2)$ form to represent the NE of arbitrary order. This compact representation and the associated expressions are the key to the development of recursive algorithms. Using the Levinson principle for all the minor subsystems we develop a

Manuscript received March 3, 1989; revised February 5, 1990. The work of T. J. Ulrych was supported by NSERC Grant 67-1804

M. Porsani is with the Universidade Federal da Bahia, PPPG/UFBA, Salvador, Bahia, Brazil

T. J. Ulrych is with the Universidade Federal da Bahia, PPPG/UFBA, Salvador, Bahia, Brazil, on leave from the Department of Geophysics and Astronomy, University of British Columbia, Vancouver, B.C. Canada V6T IW5

IEEE Log Number 9041156
Levinson-type approach for the solution of systems of equation which do not necessarily possess the Toeplitz structure. In keeping with the LR, the solution is obtained without need of the explicit knowledge of the inverse of the coefficient matrix. The methodology for the solution of NE developed in this paper is applied to the solution of NE with a coefficient matrix which possesses various symmetries. Specifically, we first consider the solution of the NE when the coefficient matrix is the doubly symmetric non-Toeplitz covariance matrix which is the case in the spectral estimation approach suggested in [8]. Next we develop an efficient algorithm for the solution of extended YuleWalker equations, a nonsymmetric Toeplitz form. Finally, we apply our approach to illustrate the solution of the well-known prediction error problem where the matrix is symmetric Toeplitz. Appendix C gives Fortran listings of the subroutines described in this paper.

\section{THEORY}

\section{A. Compact Form and Recursive Expressions}

We consider the set of overdetermined linear equations expressed by

$$
\boldsymbol{X}_{1, n} \boldsymbol{h}_{n} \approx \boldsymbol{d}
$$

where $X_{1, n}$ is any $m \times n, m \geq n$, nonsingular matrix and $d$ is the known $(m \times 1)$ data vector. The required solution is the $(n$ $\times 1)$ vector $h_{n}=\left(h_{1, n}, h_{2, n}, \cdots, h_{n, n}\right)^{T}$. The subscript $n$ designates the number of columns of $\boldsymbol{X}$ which have been used in the solution. In general we define $\boldsymbol{X}_{i, j}=\left(\boldsymbol{x}_{i}, \boldsymbol{x}_{i+1}, \cdots, \boldsymbol{x}_{j}\right)$.

The expression for the quadratic form, ' $Q_{h, j}$, associated with the least squares solution which is based on the $j$ columns of the matrix $X_{1, j}, j \leq m$, is

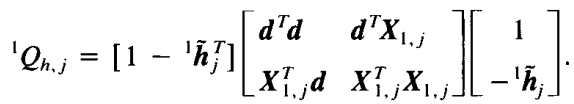

In keeping with the spirit of the Wiener estimation problem which makes use of the LR, we designate $\left(1-{ }^{\prime} \tilde{\boldsymbol{h}}_{j}^{T}\right)$ as the modeling error operator (MEO) of order $j$, and, for the sake of simplicity, we let $-{ }^{1} \tilde{h}_{j}={ }^{1} \boldsymbol{h}_{j}$. The superscript " 1 "' is required to differentiate between solutions of minor subsystems as explained below. For the complete solution we will have $\boldsymbol{h}_{n} \equiv$ ${ }^{1} h_{n}$. In all that follows, the subscript $h$ refers to quantities associated with the forward solution and the subscript $f$ refers to quantities associated with the backward solution.

Minimizing (2) and grouping the NE into an expression for the minimum ${ }^{1} Q_{h, j}={ }^{1} E_{h, j}$, we obtain the NE in expanded form as

$$
\left[\begin{array}{ll}
\boldsymbol{d}^{T} \boldsymbol{d} & \boldsymbol{d}^{T} \boldsymbol{X}_{1, j} \\
\boldsymbol{X}_{1, j}^{T} \boldsymbol{d} & \boldsymbol{X}_{1, j}^{T} \boldsymbol{X}_{1, j}
\end{array}\right]\left[\begin{array}{l}
1 \\
{ }^{1} \boldsymbol{h}_{j}
\end{array}\right]=\left[\begin{array}{l}
{ }^{1} E_{h, j} \\
\mathbf{0}_{j}
\end{array}\right]
$$

where $0_{j}=\left(0_{1}, \cdots, 0_{j}\right)^{T}$. Let us assume knowledge of the forward and backward MEO's of order $j-1,\left(1^{1} h_{j-1}^{T}\right)$ and 
$\left({ }^{1} f_{j-1}^{T} 1\right)$, which are the solutions to the two subsystems of (3) expressed by

$$
\begin{aligned}
& {\left[\begin{array}{ll}
\boldsymbol{d}^{T} \boldsymbol{d} & \boldsymbol{d}^{T} \boldsymbol{X}_{1, j-1} \\
\boldsymbol{X}_{1, j-1}^{T} \boldsymbol{d} & \boldsymbol{X}_{1, j-1}^{T} \boldsymbol{X}_{1, j-1}
\end{array}\right]\left[\begin{array}{l}
1 \\
{ }^{1} \boldsymbol{h}_{j-1}
\end{array}\right]=\left[\begin{array}{l}
{ }^{1} E_{h, j-1} \\
\mathbf{0}_{j-1}
\end{array}\right]} \\
& {\left[\begin{array}{ll}
\boldsymbol{X}_{1, j-1}^{T} \boldsymbol{X}_{1, j-1} & \boldsymbol{X}_{1, j-1}^{T} \boldsymbol{x}_{j} \\
\boldsymbol{x}_{j}^{T} \boldsymbol{X}_{1, j-1} & \boldsymbol{x}_{j}^{T} \boldsymbol{x}_{j}
\end{array}\right]\left[\begin{array}{l}
{ }^{1} \boldsymbol{f}_{j-1} \\
1
\end{array}\right]=\left[\begin{array}{l}
\mathbf{0}_{j-1} \\
{ }^{1} E_{f, j-1}
\end{array}\right] .}
\end{aligned}
$$

Applying Levinson's basic principle, where we wish to emphasize that the coefficient matrix is no longer necessarily Toeplitz, we establish the linear relationship between the MEO's of order $j-1$ and $j$, as follows:

$$
\left[\begin{array}{l}
1 \\
{ }^{1} \boldsymbol{h}_{j}
\end{array}\right]=\left[\begin{array}{l}
1 \\
{ }^{1} \boldsymbol{h}_{j-1} \\
0
\end{array}\right]+{ }^{1} h_{j, j}\left[\begin{array}{l}
0 \\
{ }^{1} \boldsymbol{f}_{j-1} \\
1
\end{array}\right] .
$$

Substituting (6) in (2) and using (4) and (5) we obtain the simplified form for ${ }^{1} Q_{h, j}$ as a function of ${ }^{1} h_{j, j}$ written as

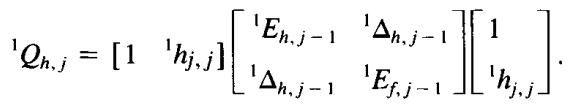

The coefficient ${ }^{1} \Delta_{h, j-1}$ in (7) is obtained using

$$
{ }^{1} \Delta_{h, j-1}=\left[\begin{array}{ll}
x_{j}^{T} d & x_{j}^{T} X_{1, j-1}
\end{array}\right]\left[\begin{array}{l}
1 \\
{ }^{1} h_{j-1}
\end{array}\right]
$$

or

$$
{ }^{1} \Delta_{h, j-1}=\left[\begin{array}{ll}
\boldsymbol{d}^{T} \boldsymbol{X}_{1, j-1} & \boldsymbol{d}^{T} \boldsymbol{x}_{j}
\end{array}\right]\left[\begin{array}{l}
{ }^{1} \boldsymbol{f}_{j-1} \\
1
\end{array}\right] .
$$

Minimizing ${ }^{1} Q_{h, j}$ in (7) with respect to ${ }^{1} h_{j, j}$ and expanding, we obtain [9]

$$
\left[\begin{array}{cc}
{ }^{1} E_{h, j-1} & { }^{1} \Delta_{h, j-1} \\
{ }^{1} \Delta_{h, j-1} & 1 \\
E_{f, j-1}
\end{array}\right]\left[\begin{array}{l}
1 \\
{ }^{1} h_{j, j}
\end{array}\right]=\left[\begin{array}{l}
{ }^{1} E_{h, j} \\
0
\end{array}\right] .
$$

This simplified representation of (2) is the heart of all of the algorithms (for example, [2]-[6]) which use Levinson's basic principle to construct the solution of order $j$ based on the solution of order $j-1$. From (9) we immediately obtain

$$
\begin{aligned}
{ }^{1} h_{j, j} & =-\frac{{ }^{1} \Delta_{h, j-1}}{{ }^{\mathrm{t}} E_{f, j-1}} \\
{ }^{1} E_{h, j} & ={ }^{1} E_{h, j-1}+{ }^{1} h_{j, j}{ }^{1} \Delta_{h, j-1} .
\end{aligned}
$$

When the coefficient matrix is equivalent to the symmetric Toeplitz autocorrelation matrix, the above expressions become equal to those resulting from the Levinson recursion [10].

Using the same principle, only now in order to obtain the backward MEO $\left({ }^{1} f_{j}^{T} 1\right)$, we write the relationship as follows:

$$
\left[\begin{array}{l}
{ }^{1} \boldsymbol{f}_{j} \\
1
\end{array}\right]=\left[\begin{array}{l}
0 \\
{ }^{2} \boldsymbol{f}_{j-1} \\
1
\end{array}\right]+{ }^{\prime} f_{j, j}\left[\begin{array}{l}
1 \\
{ }^{2} \boldsymbol{h}_{j-1} \\
0
\end{array}\right]
$$

where ${ }^{2} \boldsymbol{f}_{j-1}$ and ${ }^{2} \boldsymbol{h}_{j-1}$ are obtained as the solutions of (13) and (14) expressed below:

$$
\left[\begin{array}{ll}
\boldsymbol{X}_{2, j}^{T} \boldsymbol{X}_{2, j} & \boldsymbol{X}_{2, j}^{T} \boldsymbol{x}_{j+1} \\
\boldsymbol{x}_{j+1}^{T} \boldsymbol{X}_{2, j} & \boldsymbol{x}_{j+1}^{T} \boldsymbol{x}_{j+1}
\end{array}\right]\left[\begin{array}{l}
{ }^{2} \boldsymbol{f}_{j-1} \\
1
\end{array}\right]=\left[\begin{array}{l}
\mathbf{0}_{j-1} \\
{ }^{2} E_{f, j-1}
\end{array}\right]
$$

and

$$
\left[\begin{array}{ll}
\boldsymbol{x}_{1}^{T} \boldsymbol{x}_{1} & \boldsymbol{x}_{1}^{T} \boldsymbol{X}_{2, j} \\
\boldsymbol{X}_{2, j}^{T} \boldsymbol{x}_{1} & \boldsymbol{X}_{2, j}^{T} \boldsymbol{X}_{2, j}
\end{array}\right]\left[\begin{array}{l}
1 \\
{ }^{2} \boldsymbol{h}_{j-1}
\end{array}\right]=\left[\begin{array}{l}
{ }^{2} E_{h, j-1} \\
\mathbf{0}_{j-1}
\end{array}\right] .
$$

In a manner analogous to obtaining (9) we obtain a form for the backward MEO and we can immediately obtain expressions for ${ }^{1} f_{j, j}$ and ${ }^{1} E_{f, j}$ analogous to expressions (10) and (11). We proceed recursively to obtain the full solution ${ }^{1} \boldsymbol{h}_{n}$.

\section{B. Algorithm for Solving the NE}

We present in this section details of the algorithm which solves the NE described by (3). Using the basic methodology of decomposing our system of equations into subsystems of lesser order and coupled with the compact form for the forward and backward MEO's, we can establish a recursive Levinsontype procedure for the solution. In order to illustrate the general recursion we begin with a system of order 2 as illustrated below. We designate $\boldsymbol{d}^{T} \boldsymbol{d}$ by $a$, elements of the vector $\boldsymbol{d}^{T} \boldsymbol{X}_{i, j}$ by $b_{k}$ and elements of the matrix $\boldsymbol{X}_{i, j}^{T} \boldsymbol{X}_{i, j}$ by $s_{p, q}$. Consequently

$$
\left[\begin{array}{lll}
a & b_{1} & b_{2} \\
b_{1} & s_{1,1} & s_{1,2} \\
b_{2} & s_{2,1} & s_{2,2}
\end{array}\right]\left[\begin{array}{l}
1 \\
{ }^{1} h_{2,1} \\
{ }^{1} h_{2,2}
\end{array}\right]=\left[\begin{array}{l}
{ }^{2} E_{h, 2} \\
0 \\
0
\end{array}\right] .
$$

Assuming knowledge of $\left(1^{1} h_{1,1}\right)$ and $\left({ }^{1} f_{1,1} 1\right)$ which are the solutions of the forward and backward subsystems expressed by

$$
\left[\begin{array}{ll}
a & b_{1} \\
b_{1} & s_{1,1}
\end{array}\right]\left[\begin{array}{l}
1 \\
{ }^{1} h_{1,1}
\end{array}\right]=\left[\begin{array}{l}
{ }^{1} E_{h, 1} \\
0
\end{array}\right]
$$

and

$$
\left[\begin{array}{ll}
s_{1,1} & s_{1,2} \\
s_{2,1} & s_{2,2}
\end{array}\right]\left[\begin{array}{l}
{ }^{\prime} f_{1,1} \\
1
\end{array}\right]=\left[\begin{array}{l}
0 \\
{ }^{\prime} E_{f, 1}
\end{array}\right]
$$

we obtain $\left(1^{1} h_{2,1}{ }^{1} h_{2,2}\right)$ from

$$
\left[\begin{array}{l}
1 \\
{ }^{1} h_{2,1} \\
{ }^{1} h_{2,2}
\end{array}\right]=\left[\begin{array}{l}
1 \\
{ }^{1} h_{1,1} \\
0
\end{array}\right]+{ }^{1} h_{2,2}\left[\begin{array}{l}
0 \\
{ }^{1} f_{1,1} \\
1
\end{array}\right]
$$

where ${ }^{1} h_{2.2}$ is determined using the expression

${ }^{1} h_{2,2}=-\frac{{ }^{1} \Delta_{h, 1}}{{ }^{1} E_{f, 1}}=-\frac{\left[\begin{array}{ll}b_{2} & s_{2,1}\end{array}\right]\left[\begin{array}{l}1 \\ { }^{1} h_{1,1}\end{array}\right]}{\left[\begin{array}{ll}s_{2,1} & s_{2,2}\end{array}\right]\left[\begin{array}{l}{ }^{1} f_{1,1} \\ 1\end{array}\right]}=-\frac{\left[\begin{array}{ll}b_{1} & b_{2}\end{array}\right]\left[\begin{array}{l}{ }^{1} f_{1,1} \\ 1\end{array}\right]}{\left[\begin{array}{ll}s_{2,1} & s_{2,2}\end{array}\right]\left[\begin{array}{l}{ }^{1} f_{1,1} \\ 1\end{array}\right]}$.

We can now express the solution for order 3 as

$$
\left[\begin{array}{llll}
a & b_{1} & b_{2} & b_{3} \\
b_{1} & s_{1,1} & s_{1,2} & s_{1,3} \\
b_{2} & s_{2,1} & s_{2,2} & s_{2,3} \\
b_{3} & s_{3,1} & s_{3,2} & s_{3,3}
\end{array}\right]\left[\begin{array}{l}
1 \\
{ }^{1} h_{3,1} \\
{ }^{1} h_{3,2} \\
{ }^{1} h_{3,3}
\end{array}\right]=\left[\begin{array}{l}
{ }^{1} E_{h, 3} \\
0 \\
0 \\
0
\end{array}\right] .
$$


Once we know the forward MEO of order 2 we need obtain the backward MEO $\left({ }^{1} f_{2,2}{ }^{1} f_{2,1} 1\right)$ which solves the system below:

$$
\left[\begin{array}{lll}
s_{1,1} & s_{1,2} & s_{1,3} \\
s_{2,1} & s_{2,2} & s_{2,3} \\
s_{3,1} & s_{3,2} & s_{3,3}
\end{array}\right]\left[\begin{array}{l}
{ }^{1} f_{2,2} \\
{ }^{1} f_{2,1} \\
1
\end{array}\right]=\left[\begin{array}{l}
0 \\
0 \\
{ }^{\prime} E_{f, 2}
\end{array}\right] .
$$

The same procedure used to obtain the forward MEO of order 2 may now be employed such that the reverse MEO $\left({ }^{1} f_{2,2}{ }^{1} f_{2,1}\right.$ 1 ) is determined as

$$
\left[\begin{array}{l}
{ }^{1} f_{2,2} \\
{ }^{1} f_{2,1} \\
1
\end{array}\right]=\left[\begin{array}{l}
0 \\
{ }^{2} f_{1,1} \\
1
\end{array}\right]+{ }^{1} f_{2,2}\left[\begin{array}{l}
1 \\
{ }^{2} h_{1,1} \\
0
\end{array}\right]
$$

where

$$
{ }^{1} f_{2,2}=-\frac{{ }^{2} \Delta_{f, 1}}{{ }^{2} E_{h, 2}}=-\frac{\left[\begin{array}{ll}
s_{1,2} & s_{1,3}
\end{array}\right]\left[\begin{array}{l}
{ }^{2} f_{1,1} \\
1
\end{array}\right]}{\left[\begin{array}{ll}
s_{1,1} & s_{1,2}
\end{array}\right]\left[\begin{array}{l}
1 \\
{ }^{2} h_{1,1}
\end{array}\right]}=-\frac{\left[\begin{array}{ll}
s_{3,1} & s_{3,2}
\end{array}\right]\left[\begin{array}{l}
1 \\
{ }^{2} h_{1,1}
\end{array}\right]}{\left[\begin{array}{ll}
s_{1,1} & s_{1,2}
\end{array}\right]\left[\begin{array}{l}
1 \\
{ }^{2} h_{1,1}
\end{array}\right]}
$$

where $\left(1^{2} h_{1,1}\right)$ and $\left({ }^{2} f_{1,1} 1\right)$ are the forward and backward MEO's corresponding to the subsystem equations

$$
\left[\begin{array}{ll}
s_{1,1} & s_{1,2} \\
s_{2,1} & s_{2,2}
\end{array}\right]\left[\begin{array}{l}
1 \\
{ }^{2} h_{1,1}
\end{array}\right]=\left[\begin{array}{l}
{ }^{2} E_{h, 1} \\
0
\end{array}\right]
$$

and

$$
\left[\begin{array}{ll}
s_{2,2} & s_{2,3} \\
s_{3,2} & s_{3,3}
\end{array}\right]\left[\begin{array}{l}
{ }^{2} f_{1,1} \\
1
\end{array}\right]=\left[\begin{array}{l}
0 \\
{ }^{2} E_{f, 1}
\end{array}\right] .
$$

Knowing the forward and backward MEO's of order 2, (1 $\left.{ }^{1} h_{2,1}{ }^{1} h_{2,2}\right)$ and $\left({ }^{1} f_{2,2}{ }^{1} f_{2,1} 1\right)$, we determine ${ }^{1} h_{3,3}$ using (10) and obtain ( $1^{1} h_{3}^{T}$ ) using (6) for $j=3$. The schematic representation for the coefficients ${ }^{k} h_{j, j}$ and ${ }^{k} f_{j, j}$ is shown below:

$$
{ }^{2} f_{1,1}{ }^{2} h_{1,1}{ }^{1} f_{2,2} 7^{1} h_{3,3}
$$

Following this development for order 4 we obtain schematic representation for the coefficients ${ }^{k} h_{j, j}$ and ${ }^{k} f_{j, j}$ which follow the structure:

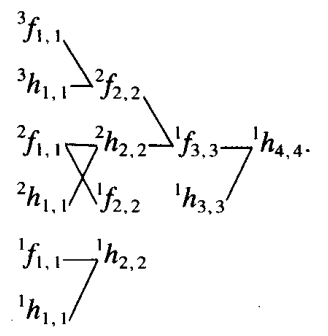

For order 5 we have

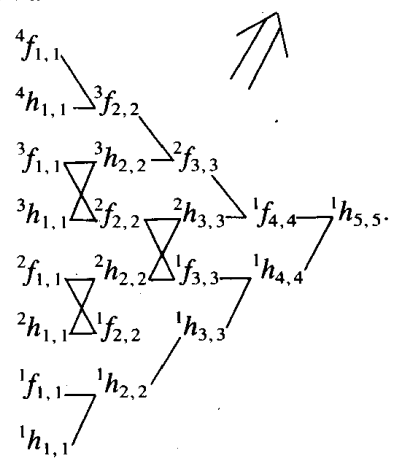

The large arrow in the diagram above indicates the direction of growth of the structure.

The coefficients ${ }^{k} h_{1,1}$ and ${ }^{k} f_{1,1}$ are related to the solutions of the forward and backward systems of order 2 which occur along the diagonal of the expanded form of the NE:

$$
\left[\begin{array}{llllll}
a & b_{1} & & & & \\
b_{1} & s_{1,1} & s_{1,2} & & & \\
& s_{2,1} & s_{2,2} & s_{2,3} & & \\
& & s_{3,2} & s_{3,3} & s_{3,4} & \\
& & & s_{4,3} & s_{4,4} & s_{4,5} \\
& & & & s_{5,4} & s_{5,5}
\end{array}\right] .
$$

In the same manner the coefficients ${ }^{k} h_{2,2}$ and ${ }^{k} f_{2,2}$ correspond to subsystems of order 3 :

$$
\left[\begin{array}{llllll}
a & b_{1} & b_{2} & & & \\
b_{1} & s_{1,1} & s_{1,2} & s_{1,3} & & \\
b_{2} & s_{2,1} & s_{2,2} & s_{2,3} & s_{2,4} & \\
& s_{3,1} & s_{3,2} & s_{3,3} & s_{3,4} & s_{3,5} \\
& & s_{4,2} & s_{4,3} & s_{4,4} & s_{4,5} \\
& & & s_{5,3} & s_{5,4} & s_{5,5}
\end{array}\right] .
$$

We note that equal subsystems imply that the solutions are necessarily equal. The number of multiplications and divisions $N_{m d}$, which are additionally required to increase the order $j$ to $j$ +1 is of order $j^{2}$. The total number of operations for a system of order $n$ is $N_{m d}=n^{3}-2 n^{2}+4 n$.

\section{The Inverse and the Determinant of the Coefficient Matrix}

We have presented a method of solving the NE which, in keeping with the Levinson principle, does not require the knowledge of either the inverse or of the determinant of the coefficient matrix which we will denote by $S_{f, n}$. In this section we would like to show that the inverse of the coefficient matrix can be expressed as a function of the reverse MEO's ( $\left.{ }^{1} f_{j}^{T} 1\right) j$ $=1, \cdots, n-1$. The procedure which follows is an extension of the approach presented by Burg [3] for the case where the coefficient matrix is symmetric Toeplitz. Given the reverse 
MEO's we can write

$$
\begin{aligned}
& {\left[\begin{array}{ll}
1 & \mathbf{0}_{n-1}^{T}
\end{array}\right] \boldsymbol{S}_{f, n}\left[\begin{array}{l}
1 \\
\mathbf{0}_{n-1}
\end{array}\right]={ }^{1} E_{f, 0}=s_{1,1}} \\
& {\left[\begin{array}{lll}
{ }^{1} f_{1,1} & 1 & \mathbf{0}_{n-2}^{T}
\end{array}\right] S_{f, n}\left[\begin{array}{l}
{ }^{1} f_{1,1} \\
1 \\
\mathbf{0}_{n-2}
\end{array}\right]={ }^{1} E_{f, 1}} \\
& {\left[\begin{array}{ll}
{ }^{1} f_{n-1}^{T} & 1
\end{array}\right] S_{f, n}\left[\begin{array}{l}
{ }^{1} f_{n-1} \\
1
\end{array}\right]={ }^{1} E_{f, n-1} .}
\end{aligned}
$$

The above equations may be combined into a matrix form as follows:

$$
\begin{aligned}
& {\left[\begin{array}{cccc}
1 & & & \\
{ }^{1} f_{1,1} & 1 & 0 & \\
\vdots & & \ddots & \\
{ }^{1} f_{n-1}^{T} & & & 1
\end{array}\right] S_{f, n}\left[\begin{array}{ccccc}
1 & & { }^{1} f_{1,1} & \cdots & { }^{1} f_{n-1} \\
& & 1 & & \\
& 0 & & \ddots & \\
& & & & 1
\end{array}\right]}
\end{aligned}
$$

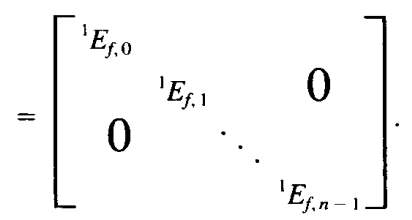

Letting $\boldsymbol{F}_{n}^{T}$ represent the matrix which premultiplies $\boldsymbol{S}_{f, n}$ and letting $\boldsymbol{E}_{n}$ represent the diagonal matrix, we obtain

$$
\boldsymbol{F}_{n}^{T} \boldsymbol{S}_{f, n} \boldsymbol{F}_{n}=\boldsymbol{E}_{n}
$$

and consequently

$$
S_{f, n}=\left[\boldsymbol{F}_{n}^{T}\right]^{-1} E_{n}\left[F_{n}\right]^{-1} .
$$

Taking the inverse in (16)

$$
\left[\boldsymbol{S}_{f, n}\right]^{-1}=\boldsymbol{F}_{n}\left[\boldsymbol{E}_{n}\right]^{-1} \boldsymbol{F}_{n}^{T} .
$$

Considering the fact that $\operatorname{det}\left[F_{n}\right]=1$ we may obtain the determinant from (16) as

$$
\operatorname{det}\left[\boldsymbol{S}_{f, n}\right]=\operatorname{det}\left[\boldsymbol{E}_{n}\right]=\prod_{j=0}^{n-1}{ }^{1} E_{f, j}
$$

Appendix A presents a numerical example of the procedure outlined above for a $(3 \times 3)$ symmetric non-Toeplitz matrix.

\section{Applications}

In this section we illustrate our general LR approach by considering three examples in which the coefficient matrix exhibits different structures.

\section{A. Normal Equations with Doubly Symmetric Non-Toeplitz Covariance Matrices}

The general expressions which we have derived take on a particularly simple form when the coefficient matrix possesses a doubly symmetric structure. In this section we explore the solution of NE when the matrix is the doubly symmetric nonToeplitz covariance matrix which arises in the forward and backward least squares method of spectral analysis proposed in [8].

We represent the expanded form of the NE of order $j$ as

$$
\left[\begin{array}{lll}
s_{h, 0} & s_{h, j-1}^{T} & s_{h, j} \\
s_{h, j-1} & S_{f, j-1} & J_{j-1} s_{h, j-1} \\
s_{h, j} & s_{h, j-1}^{T} J_{j-1} & s_{h, 0}
\end{array}\right]\left[\begin{array}{l}
1 \\
{ }^{1} h_{j}
\end{array}\right]=\left[\begin{array}{l}
{ }^{1} E_{h, j} \\
\mathbf{0}_{j}
\end{array}\right]
$$

where

$$
J_{j-1}=\left[\begin{array}{lll}
0 & & 1 \\
& 1 & \\
1 & & 0
\end{array}\right]_{j-1 \times j-1}
$$

is the reverse identity matrix of order $j-1$ and $S_{f, j-1}$ are doubly symmetric non-Toeplitz covariances matrices.

The relationship between solutions of order $j$ and $j-1$ for doubly symmetric systems results in

$$
\left[\begin{array}{l}
1 \\
\boldsymbol{h}_{j}
\end{array}\right]=\left[\begin{array}{l}
1 \\
\boldsymbol{h}_{j-1} \\
0
\end{array}\right]+h_{j, j}\left[\begin{array}{l}
0 \\
\boldsymbol{J}_{j-1} \boldsymbol{h}_{j-1} \\
1
\end{array}\right]
$$

and the compact form of the NE becomes

$$
\left[\begin{array}{cc}
{ }^{1} E_{h, j-1} & { }^{1} \Delta_{h, j-1} \\
{ }^{1} \Delta_{h, j-1} & { }^{1} E_{h, j-1}
\end{array}\right]\left[\begin{array}{l}
1 \\
{ }^{1} h_{j, j}
\end{array}\right]=\left[\begin{array}{l}
{ }^{1} E_{h, j} \\
0
\end{array}\right]
$$

with

$$
{ }^{\prime} \Delta_{h, j-1}=\left[\begin{array}{ll}
s_{h, j} & \boldsymbol{s}_{h, j-1}^{T} \boldsymbol{J}_{j-1}
\end{array}\right]\left[\begin{array}{l}
1 \\
{ }^{1} \boldsymbol{h}_{j-1}
\end{array}\right]
$$

and

$$
{ }^{1} E_{h, j}={ }^{1} E_{h, j-1}\left(1-{ }^{1} h_{j, j}^{2}\right) .
$$

Following the procedure presented earlier we may determine solutions for each subsystem of (18). The schematic representation for the coefficients ${ }^{k} h_{j, j}$ and ${ }^{k} f_{j, j}$ for order 5 is shown below:

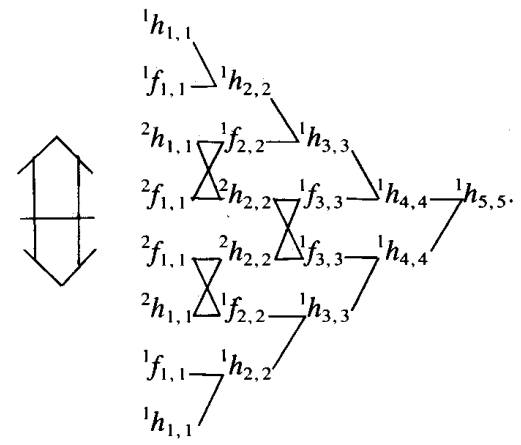


We remark that the axial symmetry in the diagram is the result of the double symmetry of the expanded form of the NE.

\section{B. Nonsymmetric Toeplitz Systems}

Extended Yule-Walker equations, where the coefficient matrix is Toeplitz but not symmetric, are of central importance in time series modeling [11]. Specifically, most algorithms for determining autoregressive-moving-average (ARMA) models rely on the fact that the AR and MA coefficients decouple depending on the order of the MA component. This decoupling is expressed by the nonsymmetric Toeplitz covariance matrix. The LR approach was extended to the solution of equations containing nonsymmetric Toeplitz matrices by G. Rybicki. His development and algorithm are presented in [7]. Our approach and algorithm are quite different.

Consider the set of nonsymmetric Toeplitz linear equations

$$
\left[\begin{array}{cccc}
r_{k} & r_{k-1} & \cdots & r_{k-n+1} \\
r_{k+1} & r_{k} & \ddots & \vdots \\
\vdots & \ddots & \ddots & r_{k-1} \\
r_{k+n-1} & \cdots & r_{k+1} & r_{k}
\end{array}\right]\left[\begin{array}{c}
h_{n, 1} \\
\vdots \\
\vdots \\
h_{n, n}
\end{array}\right]=\left[\begin{array}{c}
y_{1} \\
\vdots \\
\vdots \\
y_{n}
\end{array}\right]
$$

where we let $\boldsymbol{y}_{n}=\left(y_{1}, y_{2}, \cdots, y_{n}\right)^{T}$ be any known vector and where $k$ is not necessarily equal to 0 . We may rewrite (23) as follows:

$$
\left[\begin{array}{ll}
-\boldsymbol{y}_{j+1} & \boldsymbol{R}_{h, j+1}
\end{array}\right]\left[\begin{array}{l}
1 \\
\boldsymbol{h}_{j+1}
\end{array}\right]=\mathbf{0}_{j+1}
$$

In this case we can abandon the superscript since subsystems of $\boldsymbol{R}_{h, j+1}$ are all equal. The Levinson relation between the forward and backward solutions of subsystems of order $j+1$ is

$$
\left[\begin{array}{l}
1 \\
\boldsymbol{h}_{j+1}
\end{array}\right]=\left[\begin{array}{l}
1 \\
\boldsymbol{h}_{j} \\
0
\end{array}\right]+h_{j+1, j+1}\left[\begin{array}{l}
0 \\
\boldsymbol{b}_{j} \\
1
\end{array}\right] .
$$

Assuming known the solution of subsystems of order $j$, the compact form for (24) may be written as

$$
\left[\begin{array}{ll}
\Delta_{h, j} & E_{b, j}
\end{array}\right]\left[\begin{array}{l}
1 \\
h_{j+1, j+1}
\end{array}\right]=0
$$

where

$$
\Delta_{h, j}=\left[\begin{array}{llll}
-y_{j+1} & r_{k+j} & \cdots & r_{k+1}
\end{array}\right]\left[\begin{array}{c}
1 \\
h_{j, 1} \\
\vdots \\
h_{j, j}
\end{array}\right]
$$

and $h_{j+1, j+1}$ is given by

$$
h_{j+1, j+1}=-\frac{\Delta_{h, j}}{E_{b, j}} .
$$

Since we need the backward solutions we initially set up the recursion to solve the forward and backward systems repre- sented below:

$$
\boldsymbol{R}_{h, j+1}\left(\left[\begin{array}{l}
1 \\
\boldsymbol{a}_{j}
\end{array}\right],\left[\begin{array}{l}
\boldsymbol{b}_{j} \\
1
\end{array}\right]\right)=\left(\left[\begin{array}{l}
E_{a, j} \\
\mathbf{0}_{j}
\end{array}\right],\left[\begin{array}{l}
\mathbf{0}_{j} \\
E_{b, j}
\end{array}\right]\right) .
$$

The Levinson relations for forward and backward solutions of order $j$ are

$$
\begin{aligned}
& {\left[\begin{array}{l}
1 \\
\boldsymbol{a}_{j}
\end{array}\right]=\left[\begin{array}{l}
1 \\
\boldsymbol{a}_{j-1} \\
0
\end{array}\right]+a_{j, j}\left[\begin{array}{l}
0 \\
\boldsymbol{b}_{j-1} \\
1
\end{array}\right]} \\
& {\left[\begin{array}{l}
\boldsymbol{b}_{j} \\
1
\end{array}\right]=\left[\begin{array}{l}
0 \\
\boldsymbol{b}_{j-1} \\
1
\end{array}\right]+b_{j, j}\left[\begin{array}{l}
1 \\
\boldsymbol{a}_{j-1} \\
0
\end{array}\right] .}
\end{aligned}
$$

Assuming that the MEO of order $j-1$ are known, we obtain the compact representation for (25)

$$
\left[\begin{array}{ll}
E_{a, j-1} & \Delta_{b, j-1} \\
\Delta_{a, j-1} & E_{b, j-1}
\end{array}\right]\left(\left[\begin{array}{l}
1 \\
a_{j, j}
\end{array}\right],\left[\begin{array}{l}
b_{j . j} \\
1
\end{array}\right]\right)=\left(\left[\begin{array}{l}
E_{a, j} \\
0
\end{array}\right],\left[\begin{array}{l}
0 \\
E_{b, j}
\end{array}\right]\right)
$$

where

$$
\begin{array}{r}
\Delta_{a, j-1}=\left[\begin{array}{llll}
r_{k+j} & r_{k+j-1} & \cdots & r_{k+1}
\end{array}\right]\left[\begin{array}{c}
1 \\
a_{j-1,1} \\
\vdots \\
a_{j-1, j-1}
\end{array}\right] \\
\Delta_{b, j-1}=\left[\begin{array}{llll}
r_{k-1} & \cdots & r_{k-j+1} & r_{k-j}
\end{array}\right]\left[\begin{array}{c}
b_{j-1, j-1} \\
\vdots \\
b_{j-1,1} \\
1
\end{array}\right] .
\end{array}
$$

It turns out that for this specific structure $E_{a, j}=E_{b, j}=E_{j}$ (Appendix B) and the coefficients $a_{j, j}$ and $b_{j, j}$ are calculated using

$$
a_{j, j}=-\frac{\Delta_{a, j-1}}{E_{j-1}}, \quad b_{j, j}=-\frac{\Delta_{b . j-1}}{E_{j-1}} .
$$

The schematic representation for the coefficients $a_{j, j}, b_{j, j}$, and $h_{j, j}$ for order 5 is presented below:

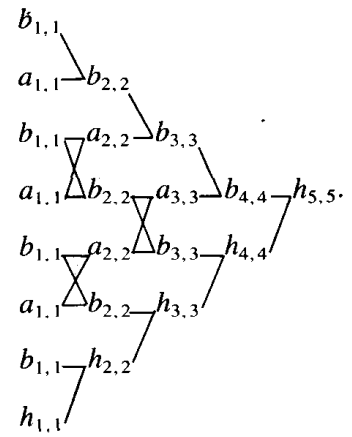


We remark that the simplification is due to the fact that subsystems of the same order are equal. In the case when the matrix $R_{h, j+1}$ is in fact symmetric, as occurs for the Wiener shaping filter, $a_{j, j}=b_{j, j}$ and the algorithm is further simplified.

Below we present the full recursion to solve $\boldsymbol{R}_{n} \boldsymbol{h}_{n}=\boldsymbol{y}_{n}$. A Fortran listing of the subroutine is given in Appendix C. Initialization:

$$
E_{0}=r_{k}, \quad \Delta_{a, 0}=r_{k+1}, \quad \Delta_{b, 0}=r_{k-1}, \quad \Delta_{h, 0}=-y_{1}
$$$$
\text { DO } j=1, n
$$

$$
\begin{gathered}
a_{j, j}=-\frac{\Delta_{a, j-1}}{E_{j-1}}, \quad b_{j, j}=-\frac{\Delta_{b, j-1}}{E_{j-1}}, \quad h_{j, j}=-\frac{\Delta_{h, j-1}}{E_{j-1}} \\
E_{j}=E_{j-1}+a_{j, j} \Delta_{b, j-1}=E_{j-1}+b_{j, j} \Delta_{a, j-1}
\end{gathered}
$$

$$
\begin{aligned}
& {\left[\begin{array}{l}
1 \\
a_{j}
\end{array}\right]=\left[\begin{array}{l}
1 \\
\boldsymbol{a}_{j-1} \\
0
\end{array}\right]+a_{j, j}\left[\begin{array}{l}
0 \\
\boldsymbol{b}_{j-1} \\
1
\end{array}\right]} \\
& {\left[\begin{array}{l}
\boldsymbol{b}_{j} \\
1
\end{array}\right]=\left[\begin{array}{l}
0 \\
\boldsymbol{b}_{j-1} \\
1
\end{array}\right]+b_{j, j}\left[\begin{array}{l}
1 \\
\boldsymbol{a}_{j-1} \\
0
\end{array}\right]} \\
& {\left[\begin{array}{l}
1 \\
\boldsymbol{h}_{j}
\end{array}\right]=\left[\begin{array}{l}
1 \\
\boldsymbol{h}_{j-1} \\
0
\end{array}\right]+h_{j, j}\left[\begin{array}{l}
0 \\
\boldsymbol{b}_{j-1} \\
1
\end{array}\right]} \\
& \Delta_{a, j}=r_{k+j+1}+\sum_{i=1}^{j} a_{j, 1} r_{k+j-i+1} \\
& \Delta_{b, j}=r_{k-j-1}+\sum_{i=1}^{j} b_{j, 1} r_{k-j+i-1} \\
& \Delta_{h, j}=-y_{j+1}+\sum_{i=1}^{j} h_{j, 1} r_{k+j-i+1}
\end{aligned}
$$

ENDDO.

$3 n^{2}+n$ multiplications and divisions are required. If it is required to solve (25) for the vector ( $1 a^{T}$ ) the above algorithm is simplified somewhat requiring only $2 n^{2}+n$ operations.

\section{The Unit Delay Prediction Error Operator}

In this particularly well-known case, the coefficient matrix is symmetric Toeplitz and we wish to very briefly illustrate how the general LR solution simplifies.

Defining $\left(1 \boldsymbol{g}_{j}^{T}\right)=\left(1, g_{j, 1}, \cdots, g_{j, j}\right)$ we may write (1) as

$$
\boldsymbol{e}_{g, j}=\boldsymbol{X}_{1, j+1}\left[\begin{array}{l}
1 \\
\boldsymbol{g}_{j}
\end{array}\right]
$$

where $e_{g, j}$ is the vector of prediction errors and

$$
\boldsymbol{X}_{1, j+1}^{T}=\left[\begin{array}{cccccccc}
x_{0} & \cdots & \cdots & x_{j} & \cdots & x_{m-1} & & 0 \\
& x_{0} & \cdots & x_{j-1} & \cdots & \cdots & x_{m-1} & \\
& & \ddots & \ddots & \ddots & \ddots & \ddots & \\
0 & & & x_{0} & \cdots & \cdots & \cdots & x_{m-1}
\end{array}\right]
$$

is the regressor matrix corresponding to the input trace $x_{t}$ of length $m$. The corresponding NE (equivalent to (3) in this case), where the superscript is not required since minor subsystems are all equal, are

$$
\boldsymbol{R}_{g, j+1}\left[\begin{array}{l}
1 \\
\mathbf{g}_{j}
\end{array}\right]=\left[\begin{array}{ll}
r_{0} & \boldsymbol{r}_{x x, j}^{T} \\
\boldsymbol{r}_{x x, j} & \boldsymbol{R}_{g, j}
\end{array}\right]\left[\begin{array}{l}
1 \\
\mathbf{g}_{j}
\end{array}\right]=\left[\begin{array}{l}
E_{g, j} \\
\mathbf{0}_{j}
\end{array}\right]
$$

where

$$
\begin{aligned}
r_{0} & =\sum_{t=0}^{m-1} x_{t}^{2} \quad \text { is the energy of } x_{t} \\
r_{x x, j} & =\left(r_{1}, \cdots, r_{j}\right)^{T} \quad \text { is the autocorrelation of } \boldsymbol{x}_{t} \\
r_{k} & =\sum_{t=0}^{m-1} x_{t} x_{t-k} \\
\boldsymbol{R}_{g, j} & =\boldsymbol{X}_{1, j}^{T} \boldsymbol{X}_{1, j} \quad \text { and is symmetric Toeplitz. }
\end{aligned}
$$

The equivalent compact form of the expanded NE is

$$
\left[\begin{array}{ll}
E_{g, j-1} & \Delta_{g, j-1} \\
\Delta_{g, j-1} & E_{g, j-1}
\end{array}\right]\left[\begin{array}{l}
1 \\
g_{j, j}
\end{array}\right]=\left[\begin{array}{l}
E_{g, j} \\
0
\end{array}\right] .
$$

Since the Toeplitz symmetry implies that all minor subsystems are equal, we may immediately obtain the Levinson recursive relationships. From (28) we obtain $g_{j, j}$ as

$$
g_{j, j}=-\frac{\Delta_{g, j-1}}{E_{g, j-1}}
$$

where

$$
\Delta_{g, j-1}=r_{x x, j}^{T} J_{j}\left[\begin{array}{l}
1 \\
g_{j-1}
\end{array}\right] .
$$

We now simply update the error energy by

$$
E_{g, j}=E_{g, j-1}\left(1-g_{j, j}^{2}\right)
$$

and we compute the full solution in the traditional manner

$$
\left[\begin{array}{l}
1 \\
g_{j}
\end{array}\right]=\left[\begin{array}{l}
1 \\
g_{j-1} \\
0
\end{array}\right]+g_{j, j}\left[\begin{array}{l}
0 \\
J_{j-1} g_{j-1} \\
1
\end{array}\right] .
$$

We remark at this stage that adding or subtracting $\boldsymbol{E}_{g, j-1}$ to $\Delta_{g, j-1}$ allows us to write

$$
\Delta_{g, j-1} \pm E_{g, j-1}=\left(r_{0}, r_{1}, \cdots, r_{j}\right)\left[\begin{array}{l}
1 \\
\boldsymbol{g}_{j-1} \pm \boldsymbol{J}_{j-1} \boldsymbol{g}_{j-1} \\
1
\end{array}\right]
$$

The symmetry, in the case of addition, and the antisymmetry, in the case of subtraction, of the term in brackets, may be utilized to reduce the number of multiplications necessary to obtain $\Delta_{g, j-1}$. This is the basic idea behind the split-Levinson algorithm [12]. The schematic representation for the reflection 
coefficients for order 5 is

$$
\begin{array}{lllll}
a_{1,1} & & & & \\
a_{1,1} & a_{2,2} & & & \\
a_{1,1} & a_{2,2} & a_{3,3} & & \\
a_{1,1} & a_{2,2} & a_{3,3} & a_{4,4} & a_{5,5} . \\
a_{1,1} & a_{2,2} & a_{3,3} & a_{4,4} & \\
a_{1,1} & a_{2,2} & a_{3,3} & & \\
a_{1,1} & a_{2,2} & & & \\
a_{1,1} & & & &
\end{array}
$$

In this particular case, due to the symmetric Toeplitz structure of the coefficient matrix, we obtain the simplest possible form in the diagramatic representation of the recursion.

\section{Discussion}

We have presented a Levinson-type approach to the solution of NE where the coefficient matrix need not have the Toeplitz structure. The methodology which has been presented reduces to LR when the coefficient matrix is symmetric Toeplitz. In the case where the matrix is partially Toeplitz, the approach makes use of LR inasmuch as the partially Toeplitz structure permits.

Another interesting aspect of the method is that, in a manner similar to the growth of crystals, the structure formed by the series of the coefficients ${ }^{h} h_{j, j},{ }^{k} f_{j, j}$ augments with the addition of new coefficients to the already existing structure. This property implies that the computation of the solution for order $j+$ 1 is not a separate problem, requiring only $j^{2}$ additional operations to the solution for order $j$. This interesting property is of great practical value when we wish to solve a system of NE of the same order $n$ which contains a common nucleus. In the case when the only difference between them is the last line and column, once that one of the NE is resolved, the remainder may be resolved using approximately $n^{2}$ operations. For all minor subsystems the values of ${ }^{k} E_{h, j-1}$ and ${ }^{k} E_{f, j-1}$, present in the denominator of the coefficients ${ }^{k} f_{j, j}$ and ${ }^{k} h_{j, j}$, supply information about the linear independence of the traces and consequently allow a measure of the relative importance of each vector in the modeling.

The presence of a great number of zeros in the coefficient matrix may also be utilized in our approach by omitting unnecessary multiply and add operations. As an example, considering tridiagonal matrices, only subsystems of order 2 along the principal diagonal possess nonzero elements and only a single multiply and add operation is required for the computation of the quantities ${ }^{k} \Delta_{h, j-1},{ }^{k} \Delta_{f, j-1},{ }^{k} E_{h, j-1}$ and ${ }^{k} E_{f, j-1}$.

Finally we wish to point out that the use of the expressions for ${ }^{k} \Delta_{h, j-1}$ and ${ }^{k} \Delta_{f, j-1}$ in the algorithm which we have described makes the method also applicable to the solution of nonsymmetric systems. In this case $S_{n}$ corresponds to the nonsymmetric coefficient matrix, the vector $\boldsymbol{b}_{n}$ corresponds to the righthand side vector of the equations which are to be solved and $\tilde{h}_{n}$ $=-\boldsymbol{h}_{\boldsymbol{n}}$ will become the solution vector.

Inasmuch as LR is not only an important time saving algorithm but also reflects, for example, the physical aspect of the problem of the reflection of acoustic energy in a plane layered earth, the extension of this approach to other than symmetric Toeplitz systems may find application in the modeling of physical problems.

\section{APPENDIX A}

In this Appendix we present a small example illustrating the steps in the algorithm and the computation of the determinant and the inverse.

Consider the expanded NE presented below:

$$
\left[\begin{array}{rrrr}
15 & -2 & 1 & 4 \\
-2 & 7 & 1 & 2 \\
1 & 1 & 18 & 6 \\
4 & 2 & 6 & 6
\end{array}\right]\left[\begin{array}{l}
1 \\
h_{3,1} \\
h_{3,2} \\
h_{3,3}
\end{array}\right]=\left[\begin{array}{l}
E_{h, 3} \\
0 \\
0 \\
0
\end{array}\right] .
$$

The corresponding coefficient diagram and the expressions for the coefficients ${ }^{k} f_{j, j}$ and ${ }^{k} h_{j, j}$, becomes

$$
\begin{aligned}
& { }^{2} f_{1,1}=-\frac{1}{3} \\
& { }^{2} h_{1,1}=-\frac{1}{18} \quad{ }^{1} f_{2,2}=-\frac{\left[\begin{array}{ll}
2 & 1
\end{array}\right]\left[\begin{array}{c}
1 \\
-1 / 3
\end{array}\right]}{\left[\begin{array}{ll}
1 & 7
\end{array}\right]\left[\begin{array}{c}
-1 / 18 \\
1
\end{array}\right]}=-\frac{6}{25} \\
& { }^{\prime} h_{3,3}=-\frac{\left[\begin{array}{lll}
4 & 2 & 6
\end{array}\right]\left[\begin{array}{l}
1 \\
h_{2,1} \\
h_{2,2}
\end{array}\right]}{\left[\begin{array}{lll}
2 & 6 & 6
\end{array}\right]\left[\begin{array}{c}
f_{2,2} \\
f_{2,1} \\
1
\end{array}\right]} \\
& { }^{1} f_{1,1}=-\frac{1}{7} \quad{ }^{1} h_{2,2}=-\frac{\left[\begin{array}{ll}
1 & 1
\end{array}\right]\left[\begin{array}{l}
1 \\
2 / 7
\end{array}\right]}{\left[\begin{array}{ll}
1 & 18
\end{array}\right]\left[\begin{array}{c}
-1 / 7 \\
1
\end{array}\right]}=-\frac{9}{125} \\
& { }^{1} h_{1,1}=\frac{2}{7}
\end{aligned}
$$

where

$$
\begin{aligned}
& {\left[\begin{array}{l}
1 \\
h_{2,1} \\
h_{2,2}
\end{array}\right]=\left[\begin{array}{l}
1 \\
2 / 7 \\
0
\end{array}\right]-\frac{9}{125}\left[\begin{array}{l}
0 \\
1 / 7 \\
1
\end{array}\right]=\left[\begin{array}{c}
1 \\
0.0296 \\
-0.072
\end{array}\right]} \\
& {\left[\begin{array}{l}
f_{2,2} \\
f_{2,1} \\
1
\end{array}\right]=\left[\begin{array}{l}
0 \\
1 / 3 \\
1
\end{array}\right] \frac{6}{25}\left[\begin{array}{l}
1 \\
1 / 18 \\
0
\end{array}\right]=\left[\begin{array}{c}
-0.24 \\
-0.32 \\
1
\end{array}\right]}
\end{aligned}
$$


and

$$
\begin{aligned}
{\left[\begin{array}{l}
1 \\
h_{3,1} \\
h_{3,2} \\
h_{3,3}
\end{array}\right] } & =\left[\begin{array}{c}
1 \\
0.296 \\
-0.072 \\
0
\end{array}\right]-\frac{4.16}{36}\left[\begin{array}{c}
0 \\
-0.24 \\
-0.32 \\
1
\end{array}\right] \\
& =\left[\begin{array}{c}
1 \\
0.573333 \\
0.297776 \\
-1.155556
\end{array}\right] .
\end{aligned}
$$

The determinant and the inverse of the coefficient matrix depend on the reverse MEO's and on the quantities ${ }^{k} E_{f, j}$

$$
\begin{aligned}
& { }^{\mathrm{I}} E_{f, 0}=7 \\
& { }^{1} E_{f, 1}=\left[\begin{array}{ll}
1 & 18
\end{array}\right]\left[\begin{array}{l}
1 / 7 \\
1
\end{array}\right]=\frac{125}{7} \\
& { }^{1} E_{f, 2}=\left[\begin{array}{lll}
2 & 6 & 6
\end{array}\right]\left[\begin{array}{c}
-0.24 \\
-0.32 \\
1
\end{array}\right]=\frac{18}{5} .
\end{aligned}
$$

The determinant is now obtained as

$$
\operatorname{det}\left[S_{f, 3}\right]=\operatorname{det}\left[\begin{array}{rrr}
7 & 1 & 2 \\
1 & 18 & 6 \\
2 & 6 & 6
\end{array}\right]={ }^{1} E_{f, 0}{ }^{1} E_{f, 1}{ }^{1} E_{f, 2}=450
$$

and the matrix inverse as

$$
\begin{aligned}
{\left[S_{f, 3}\right]^{-1}=} & {\left[\begin{array}{lll}
1 & 1 / 7 & -0.24 \\
0 & 1 & -0.32 \\
0 & 0 & 1
\end{array}\right]\left[\begin{array}{lll}
1 / 7 & 0 & 0 \\
0 & 7 / 125 & 0 \\
0 & 0 & 5 / 18
\end{array}\right] } \\
& \cdot\left[\begin{array}{ccc}
1 & 0 & 0 \\
1 / 7 & 1 & 0 \\
-0.24 & -0.32 & 1
\end{array}\right] \\
= & {\left[\begin{array}{rrr}
0.159999 & 0.013333 & -0.066666 \\
0.013333 & 0.084444 & -0.088888 \\
-0.066666 & -0.088888 & 0.277777
\end{array}\right] . }
\end{aligned}
$$

APPENDIX B

We prove in this Appendix that $E_{a, j}=E_{b . j}$ in the LR for nonsymmetric Toeplitz matrices.

Given the system for order 1

$$
\left[\begin{array}{ll}
r_{k} & r_{k-1} \\
r_{k+1} & r_{k}
\end{array}\right]\left(\left[\begin{array}{l}
1 \\
a_{1,1}
\end{array}\right],\left[\begin{array}{l}
b_{1,1} \\
1
\end{array}\right]\right)=\left(\left[\begin{array}{l}
E_{a, 1} \\
0
\end{array}\right],\left[\begin{array}{l}
0 \\
E_{b, 1}
\end{array}\right]\right)
$$

we obtain

$$
a_{1,1}=-\frac{r_{k+1}}{r_{k}}, \quad b_{1,1}=-\frac{r_{k-1}}{r_{k}} .
$$

and

$$
E_{a, 1}=r_{k}+a_{1,1} r_{k-1}, \quad E_{b, 1}=r_{k}+b_{1,1} r_{k+1} .
$$

Substituting the expressions for $a_{1,1}$ and $b_{1,1}$ in (B.1) we immediately verify that $E_{a, 1}=E_{b, 1}$. Considering now the secondorder system

$$
\begin{gathered}
{\left[\begin{array}{lll}
r_{k} & r_{k-1} & r_{k-2} \\
r_{k+1} & r_{k} & r_{k-1} \\
r_{k+2} & r_{k+1} & r_{k}
\end{array}\right]\left(\left[\begin{array}{l}
1 \\
a_{2,1} \\
a_{2,2}
\end{array}\right],\left[\begin{array}{l}
b_{2,2} \\
b_{2,1} \\
1
\end{array}\right]\right)} \\
=\left(\left[\begin{array}{l}
E_{a, 2} \\
0 \\
0
\end{array}\right],\left[\begin{array}{l}
0 \\
0 \\
E_{b, 2}
\end{array}\right]\right)
\end{gathered}
$$

or in terms of the compact representation

$$
\left[\begin{array}{cc}
E_{a, 1} & \Delta_{b, 1} \\
\Delta_{a, 1} & E_{a, 1}
\end{array}\right]\left(\left[\begin{array}{l}
1 \\
a_{2,2}
\end{array}\right],\left[\begin{array}{l}
b_{2,2} \\
1
\end{array}\right]\right)=\left(\left[\begin{array}{l}
E_{a, 2} \\
0
\end{array}\right],\left[\begin{array}{l}
0 \\
E_{b, 2}
\end{array}\right]\right)
$$

we obtain

$$
a_{2,2}=-\frac{\Delta_{a, 1}}{E_{a, 1}}, \quad b_{2,2}=-\frac{\Delta_{b, 1}}{E_{a, 1}}
$$

and

$$
E_{a, 2}=E_{a, 1}+a_{2,2} \Delta_{b, 1}, \quad E_{b, 2}=E_{a, 1}+b_{2,2} \Delta_{a, 1} .
$$

In an analogous manner, substituting the expressions for $a_{2.2}$ and $b_{2,2}$ in (B.2) we can verify that $E_{a, 2}=E_{b, 2}$. Following this procedure it is clear that $E_{a, j}=E_{b, j}$ for all $j$.

\section{APPENDIX C}

This Appendix presents a Fortran listing of the algorithms which are discussed in the text.

SUBROUTINE SOLNE $(n, \mathrm{Ch}, h, \mathrm{Eh}, \mathrm{Ha})$$$
\text { C }
$$

C Purpose: Least squares solution of system of equations $\boldsymbol{X}_{1, n} \boldsymbol{h}_{n}=\boldsymbol{d}$

C Method: Levinson extension for symmetric non-Toeplitz normal equations

C SOLNE requires HFILT and FFILT

C Input parameters:

C n-Number of coefficients

C $\quad$ Ch - Expanded coefficient matrix

$$
\boldsymbol{C}_{h, n+1}\left[\begin{array}{l}
1 \\
h_{n}
\end{array}\right]=\left[\begin{array}{ll}
\boldsymbol{d}^{T} \boldsymbol{d} & \boldsymbol{d}^{T} \boldsymbol{X}_{1, n} \\
\boldsymbol{X}_{1, n}^{T} \boldsymbol{d} & \boldsymbol{X}_{1, n}^{T} \boldsymbol{X}_{1, n}
\end{array}\right]\left[\begin{array}{l}
1 \\
\boldsymbol{h}_{n}
\end{array}\right]=\left[\begin{array}{l}
E_{h, n} \\
0_{n}
\end{array}\right]
$$

C Output parameters:

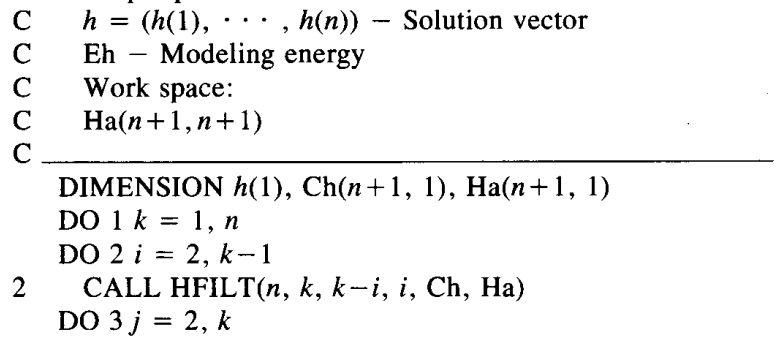


SUBROUTINE FFILT $(m, n 2, n 3, n, i, j, \mathrm{Ch}, \mathrm{Ha})$

DIMENSION $\mathrm{Ch}(m, 1), \mathrm{Ha}(m, 1)$

$\mathrm{SN}=\mathrm{Ch}(j+1, j+1+i)$

$\mathrm{SD}=\mathrm{Ch}(j+1, j+1)$

DO $1 k=2, i$

$\mathrm{SN}=\mathrm{SN}+\mathrm{Ch}(j+1, n 2-k) * \mathrm{Ha}(n 3-k, n-i+1)$

$\mathrm{SD}=\mathrm{SD}+\mathrm{Ch}(j+1, j+k) * \mathrm{Ha}(k-1, i-1)$

$\mathrm{Ha}(n-i+1, n-i)=-\mathrm{SN} / \mathrm{SD}$

DO $2 k=1, i-1$

2

$\mathrm{Ha}(m-k, n-i)=\mathrm{Ha}(m-k, n-i+1)+\mathrm{Ha}(n-i+1$, $n-i) * \mathrm{Ha}(i-k, i-1)$

\section{RETURN}

END.

SUBROUTINE $\operatorname{SOLCOV}(n, \mathrm{Ca}, a, \mathrm{Ea}, \mathrm{Ha})$

C

C Purpose: Least squares solution of forward and backward PEO

C Method: Levinson extension for doubly symmetric nonToeplitz normal equations

C SOLCOV requires FFILT and HFILT

C Input parameters:

C $n$ - Number of coefficients

$\mathrm{C} \quad \mathrm{Ca}-$ Expanded coefficient matrix

$$
C_{a, n+1}\left[\begin{array}{l}
1 \\
a_{n}
\end{array}\right]=\left[\begin{array}{l}
E_{a, n} \\
0_{n}
\end{array}\right]
$$

C Output parameters:

C $a=(1, a(1), \cdots, a(n))-$ Prediction error operator

C Ea - Forward and backward modeling energy

C Work space:

C $\mathrm{Ha}(n+1, n+1)$

C

DIMENSION $a(1), \mathrm{Ca}(n+1,1), \mathrm{Ha}(n+1,1)$ $m 1=\operatorname{INT}((n+1) / 2)$

DO $1 k=1, m 1$

DO $2 i=2, k-1$

$2 \operatorname{CALL~HFILT}(n, k, k-i, i, \mathrm{Ca}, \mathrm{Ha})$

DO $3 j=2, k$

3 CALL FFILT $(n+1, k+2, n+2, n, j-1, k-j+1, \mathrm{Ca}, \mathrm{Ha})$
$1 \quad \operatorname{CALL} \operatorname{HFILT}(n, k+1, k, 1, \mathrm{Ca}, \mathrm{Ha})$

DO $4 k=1, n-m 1$

DO $5 i=1, n-m 1-k$

$5 \quad \operatorname{CALL} \operatorname{HFILT}(n, m 1+k, m 1+k-1-i, i+1, \mathrm{Ca}, \mathrm{Ha})$

DO $6 i=1,2 * m 1-n-1+2 * k$

$6 \quad \mathrm{Ha}(n+1-i, 2 *(n-k-m 1)+1)$

$=\mathrm{Ha}(i, 2 * m 1-n-1+2 * k)$

DO $7 j=1, n-m 1-k$

$7 \quad$ CALL FFILT $(n+1, m 1+2+k, n+2, n$,

$2 * m 1-n-1+2 * k+j, n-m 1-k+1-j, \mathrm{Ca}, \mathrm{Ha})$

4 CALL HFILT $(n, m 1+k+1, m 1+k, 1, \mathrm{Ca}, \mathrm{Ha})$

$a(1)=1$

$\mathrm{Ea}=\mathrm{Ca}(1,1)$

DO $8 i=1, n$

8

$\mathrm{Ea}=\mathrm{Ea}+a(i+1) * \mathrm{Ca}(1, i+1)$

\section{RETURN}

END.

SUBROUTINE EXTYW $(n, r, y, a, b, h)$

$\mathrm{C}$ Purpose: Resolve system of non-symmetric Toeplitz equations

$\left[\begin{array}{cccc}r_{n} & r_{n-1} & \cdots & r_{1} \\ r_{n+1} & r_{n} & \cdots & r_{2} \\ \vdots & \vdots & & \vdots \\ r_{2 n-1} & & r_{n+1} & r_{n}\end{array}\right]\left[\begin{array}{c}h_{1} \\ \vdots \\ h_{n}\end{array}\right]=\left[\begin{array}{c}y_{1} \\ \vdots \\ y_{n}\end{array}\right]$

C Input parameters:

C $n$ - number of coefficients

C $\quad r-(r(1), \cdots, r(2 n-1))$ autocorrelation vector with

$2 n-1$ elements

C $\quad y$ - right-hand side of system of equations

C Output parameters:

C $h=(h(1), \cdots, h(n))-$ solution vector

C $a$ forward MEO

C $b$ backward MEO

C

DIMENSION $r(1), y(1), a(1), b(1), h(1)$

DELTa $=r(n+1)$

$\mathrm{DELTb}=r(n-1)$

DELTh $=-y(1)$

$E=r(n)$

DO $1 j=1, n$

$a(j)=-\mathrm{DELTa} / \mathrm{E}$

$b(j)=-\mathrm{DELT} b / \mathrm{E}$

$h(j)=-\mathrm{DELTh} / \mathrm{E}$

$E=E+a(j) * \mathrm{DELTb}$

DO $2 i=2, j$

$j i=j-i+1$

$h(i-1)=h(i-1)+h(j) * b(j i)$

SAVE $=a(i-1)$

$a(i-1)=a(i-1)+a(j) * b(j i)$

2

$b(j i)=b(j i)+b(j) * \mathrm{SAVE}$

IF $(j$.EQ. $n)$ RETURN

DELTa $=r(n+j+1)$

$\mathrm{DELTb}=r(n-j-1)$

DELTh $=-y(j+1)$

DO $3 i=1, j$

$j 1=j+1-i$

DELTa $=$ DELTa $+a(i) * r(n+j 1)$

$\mathrm{DELTb}=\mathrm{DELTb}+b(i) * r(n-j 1)$ 


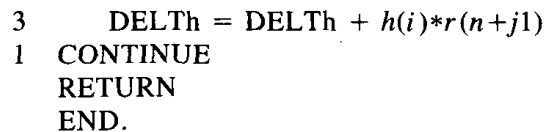

\section{ACKNOWLEDGMENT}

The authors wish to express their gratitude to Petrobras and the Conselho National de Desenvolvimento Científico e Tecnológico, CNPq, for generous support.

\section{REFERENCES}

[1] N. Levinson, "The Wiener rms (root-mean-square) error criterion in filter design and prediction," J. Math. Phys., vol. 25, pp. $261-278,1947$.

[2] S. M. Simpson, E. A. Robinson, R. A. Wiggins, and C. I. Wunsch, "Studies in optimum filtering of single and multiple stochastic processes," Massachusetts Institute of Technology, Cambridge, 1963.

[3] J. P. Burg, "Maximum entropy spectrum analysis," Ph.D. dissertation, Dep. Geophys., Stanford Univ., Stanford, CA, 1975.

[4] M. Morf, B. Dickinson, T. Kailath, and A. Vieira, "Recursive solution of covariance equations for linear prediction," IEEE Trans. Acoust., Speech, Signal Processing, vol. ASSP-25, pp. 429-433, 1977.

[5] S. L. Marple, "A new autoregressive spectrum analysis algorithm," IEEE Trans. Acoust., Speech, Signal Processing, vol. ASSP-28, pp. 441-454, 1980

[6] D. Manolakis, N. Kalonptsidis, and G. Carayannis, "Fast algorithms for discrete-time Wiener filters with optimum lag," IEEE Trans. Acoust., Speech, Signal Processing, vol. ASSP-31, pp. $168-179,1983$.

[7] W. H. Press, B. P. Flannery, S. A. Teukolsky, and W. T. Vettering, Numerical Recipes, The Art of Scientific Computing. New York: Cambridge, 1986.

[8] T. J. Ulrych and R. W. Clayton, "Time series modeling and maximum entropy," Phys. Earth Planetary Interiors, vol. 12, pp. 188-200, Aug. 1976 .

[9] M. J. Porsani and T. J. Ulrych, "Discrete convolution by means of forward and backward modeling," Trans. Acoust., Speech, Signal Processing, vol. 37, pp. 1680-1686, Nov. 1989.
[10] M. J. Porsani and W. J. Vetter, "An optimal formulation for (Levinson) recursive design of $L$-lagged minimum energy filters," in Proc. 54th Ann. Int. Soc. Exploration Geophys. (SEG) Meeting (Atlanta, GA), 1984, pp. 604-606.

[11] B. Friedlander and B. Porat, "The modified Yule-Walker method of spectral estimation," IEEE Trans. Aerosp. Electron. Syst., vol. AES-20, pp. 172, 1984.

[12] P. Delsarte and Y. V. Genin, "The split Levinson algorithm," IEEE Trans. Acoust., Speech, Signal Processing, vol. ASSP-34, pp. 470-478, June 1986.

Milton Porsani received the B.Sc. degree in geology from the University of São Paolo in 1976, the M.Sc. degree in geophysics from the University Federal do Para, and the Ph.D. degree in geophysics from the University Federal da Bahia in 1986.

From 1978 to 1982 he was with Nucleo de Ciencias Geofisicas e Geologicas (NCGG), University Federal do Para. Since 1983 he has been a Researcher with the Programa de Pesquisa e Pos-Graduacao en Geofisica PPPG/UFBA; Brazil. His research interests include timeseries analysis and inverse theory in geophysical exploration.

Dr. Porsani is a member of the Sociedade Brasileira de Geofisica (SBGF).

Tad J. Ulrych received the B.Sc. degree (honors) from the University of London in 1957, and the M.Sc. and Ph.D. degrees from the University of British Columbia in 1961 and 1963, respectively.

From 1962 to 1965 he was with the University of Western Ontario. Since 1965 he has been with the University of British Columbia. Since 1987 he has been a Visiting Professor at the Programa de Pesquisa e Pos-Graducao en Geofisica PPPG/UFBA, Brazil. His research interests include applications of time-series analysis and inverse theory to geophysical exploration.

Dr. Ulrych received the Killam Fellowship in 1983. He is a member of SEG and SEG Japan. 